\title{
Suppression of Nitric Oxide (NO)-Dependent Behavior by Double-Stranded RNA-Mediated Silencing of a Neuronal NO Synthase Gene
}

\author{
Sergei A. Korneev, Ildikó Kemenes, Volko Straub, Kevin Staras, Elena I. Korneeva, György Kemenes, \\ Paul R. Benjamin, and Michael O'Shea \\ Sussex Centre for Neuroscience, University of Sussex, Brighton BN1 9QG, United Kingdom
}

We have used double-stranded RNA (dsRNA)-mediated RNA interference (RNAi) to disrupt neuronal nitric oxide (NO) synthase (nNOS) gene function in the snail Lymnaea stagnalis and have detected a specific behavioral phenotype. The injection of whole animals with synthetic dsRNA molecules targeted to the nNOS-encoding mRNA reduces feeding behavior in vivo and fictive feeding in vitro and interferes with NO synthesis by the CNS. By showing that synthetic dsRNA targeted to the nNOS mRNA causes a significant and long-lasting reduction in the

Nitric oxide (NO) is a gaseous signaling molecule in the nervous systems of both vertebrates and invertebrates (Bredt and Snyder, 1992; Garthwaite and Boulton, 1995; Jacklet, 1997). We and others have shown that it acts as a neurotransmitter in the feeding neural network of Lymnaea stagnalis, contributing to the chemosensory initiation of feeding behavior (Moroz et al., 1993; Elphick et al., 1995). Recently we have cloned and sequenced a mRNA from Lymnaea CNS (Korneev et al., 1998) that encodes a neuronal isoform of NO synthase (Lym-nNOS). Importantly, LymnNOS mRNA is expressed in key modulatory neurons of the feeding network (Korneev et al., 1998, 1999). This indicates the possibility that a transcriptionally active nNOS gene is specifically required for feeding behavior. There is however no direct evidence to support this primarily because classical gene knock-out technologies are not presently available in molluscs. An alternative approach therefore was required to link the expression of specific genes to their effects at behavioral and cellular levels.

A recently discovered phenomenon, double-stranded RNA (dsRNA)-mediated RNA interference (RNAi), has now provided us with a potential tool for targeted gene manipulation in a genetically intractable system. It is based on the remarkable and not fully understood ability of dsRNA molecules to induce sequence-specific gene silencing in vivo (Fire et al., 1998). Here we have used RNAi to investigate the importance of Lym-nNOS gene expression in normal feeding behavior and its electrophysiological correlate in identified motoneurons of the feeding neural network. We show that the injection of whole animals with synthetic dsRNA molecules targeted to Lym-nNOS mRNA reduces feeding behavior in vivo and fictive feeding in vitro and

\footnotetext{
Received Dec. 28, 2001; revised March 5, 2002; accepted March 14, 2002.

The work was supported by a grant from the Biotechnology and Biological Sciences Research Council, UK. G.K. is a Medical Research Council Senior Fellow. Correspondence should be addressed to Michael O'Shea, Sussex Centre for Neuroscience, University of Sussex, Brighton BN1 9QG, UK. E-mail: m.oshea@sussex.ac.uk.

Copyright (C) 2002 Society for Neuroscience $\quad 0270-6474 / 02 / 220001-\bullet \$ 15.00 / 0$
}

levels of Lym-nNOS mRNA, we verify that specific RNAi has occurred. Importantly, our results establish that the expression of nNOS gene is essential for normal feeding behavior. They also show that dsRNA can be used in the investigation of functional gene expression in the context of whole animal behavior, regardless of the availability of targeted mutation technologies.

Key words: nNOS; dsRNA; RNAi; gene silencing; feeding behavior; Lymnaea stagnalis

interferes with NO synthesis by the CNS. Fully consistent with these findings, dsRNA injection causes a specific and long-lasting reduction in the levels of Lym-nNOS mRNA in the CNS. Importantly, our findings show that RNAi can be used to investigate functional gene expression in the context of whole animal behavior in a system that is particularly amenable to molecular, behavioral, and electrophysiological analysis, but in which targeted mutation technologies are not available.

\section{MATERIALS AND METHODS}

Experimental animals. Laboratory-bred stock of L. stagnalis was obtained from the Free University (Amsterdam, The Netherlands) and kept in groups in large holding tanks containing copper-free water at $18-20^{\circ} \mathrm{C}$ on a $12 \mathrm{hr}$ light/dark cycle. The animals were fed lettuce three times and a vegetable-based fish food twice per week.

Synthesis of dsRNA and single-stranded RNA. A PCR generated fragment of Lym-nNOS cDNA (positions 439-2046) was cloned into the pCRII-TOPO vector (Invitrogen, San Diego, CA). The recombinant plasmid was then digested with either BamHI or $X b a \mathrm{I}$ and used to synthesize either sense or antisense cRNAs by means of Ampliscribe T7 or Ampliscribe SP6 kits (Cambio, Cambridge, UK), respectively. Equimolar amounts of single-stranded sense [referred to later as nNOS single-stranded RNA (ssRNA)] and antisense cRNAs were hybridized at $50^{\circ} \mathrm{C}$ in a buffer containing $20 \mathrm{mM}$ HEPES, $200 \mathrm{~mm} \mathrm{KCl}$, and $1 \mathrm{~mm}$ EDTA. Typically, $>99 \%$ of the single-stranded RNA molecules were engaged in the formation of dsRNA after $10 \mathrm{hr}$ of incubation. We will

This article is published in The Journal of Neuroscience, Rapid Communications Section, which publishes brief, peerreviewed papers online, not in print. Rapid Communications are posted online approximately one month earlier than they would appear if printed. They are listed in the Table of Contents of the next open issue of JNeurosci. Cite this article as: JNeurosci, 2002, 22:RC227 (1-5). The publication date is the date of posting online at www.jneurosci.org.

http://www.jneurosci.org/cgi/content/full/6422 
refer to this dsRNA as "nNOS dsRNA." To produce a neutral control dsRNA, a transcript from a Lymnaea pseudogene was cloned into the pCRII-TOPO vector and the plasmid was used to produce dsRNA molecules $(\sim 600 \mathrm{bp}$ in length) targeted to this transcript as described above. We will refer to this dsRNA as the "unrelated dsRNA." Animals were injected with $500 \mathrm{ng}$ of dsRNA (nNOS or unrelated) or with $1 \mu \mathrm{g}$ of nNOS ssRNA dissolved in $100 \mu$ l saline or with the same volume of saline buffer only.

Behavioral experiments. Snails were starved for $24 \mathrm{hr}$ and then placed individually into Petri dishes containing $90 \mathrm{ml}$ of water. To measure their feeding response to a general disturbance caused by delivering solutions to the dish, $5 \mathrm{ml}$ of water was added to the dish and the number of "rasps" (stereotyped feeding movements of the mouth) was counted for 2 min. At the end of this period, $5 \mathrm{ml}$ of sucrose solution $(0.4 \mathrm{M}$ diluted to $0.02 \mathrm{M}$ final concentration) was added and rasps were counted for an additional 2 min. An individual feeding score was generated by subtracting the number of rasps elicited by water from that elicited by the addition of the sucrose solution. Animals were assigned to one of three or four groups matched for feeding scores (established by a one-way ANOVA). Animals in the experimental groups were each injected with nNOS dsRNA. In the first type of experiment, animals in two control groups were injected either with nNOS ssRNA or with saline. The feeding response to sucrose was retested as described above 3,24, and $48 \mathrm{hr}$ after the injections using a blind experimental protocol. In the second type of experiment, an additional control group was used in which the animals were injected with the unrelated dsRNA. The animals in this experiment were retested at $24 \mathrm{hr}$ after injection. In both types of experiments, individual feeding scores obtained in the dsRNA-, ssRNA-, or saline-injected animals were subtracted from their feeding scores obtained before injection. The difference data (a suppression of feeding score) were first subjected to an ANOVA with repeated measures $(3,24$, and $48 \mathrm{hr}$ experiments) or to a one-way ANOVA (24 hr experiment); if this revealed a source of significant difference, additional between-group comparisons were made using multiple post hoc tests (see Results).

Electrophysiological experiments. Individual feeding scores were obtained (as described above) for animals that were then injected with either the nNOS dsRNA or nNOS ssRNA (see above). Feeding responses were remeasured $48 \mathrm{hr}$ after injection and semi-intact lip-CNS in vitro preparations were set up as described previously (Kemenes et al., 2002). Intracellular recordings were made from identified motoneurons known to participate in the feeding neural network (Benjamin and Elliott, 1989), enabling fictive feeding cycles to be monitored while sucrose was superfused on the lips for $20 \mathrm{sec}$ at $0.02 \mathrm{M}$. Fictive feeding cycles, the in vitro correlate of feeding, were counted for 2 min before and after the sucrose application. The number of fictive feeding cycles in response to sucrose in each preparation was normalized to the mean number of spontaneously occurring fictive feeding cycles in the same group of preparations. This normalized data were compared between the nNOS dsRNA and ssRNA groups using unpaired $t$ tests.

Measurement of NO production. Whole CNSs isolated from the animals used in the behavioral experiments described above were used to measure NO production using the NO indicator dye 4,5-diaminofluorescein (DAF-2). Each individual CNS was incubated in a solution of DAF-2 (2 $\mu \mathrm{M})$ and L-arginine $(1 \mathrm{mM})$ in HEPES-buffered saline (total volume 1.2 $\mathrm{ml}$ ). Emission at $515 \mathrm{~nm}$ produced by an excitation wavelength of $485 \mathrm{~nm}$ was measured immediately after exposure to the dye $\left(t_{0}\right)$ and $120 \mathrm{~min}$ later $\left(t_{120}\right)$. The results were corrected for background emission by subtracting the readings obtained from samples containing all the reagents but without a CNS.

Analysis of gene expression by multiplex reverse transcription-PCR. RNA was isolated and purified from individual CNSs dissected 6 or $24 \mathrm{hr}$ after the injections using the NucleoSpin RNA II kit (Macherey-Nagel, Düren, Germany). Reverse transcription (RT) reactions were performed on each sample and $5 \mu \mathrm{l}$ of the reaction products was added to amplification mixtures containing primers specific for nNOS and $\beta$-tubulin cDNAs. After 25 cycles, PCR products were resolved on $1 \%$ agarose gel and analyzed using the Electrophoresis Documentation and Analysis System 290 (Eastman-Kodak, Rochester, NY).

\section{RESULTS}

\section{nNOS dsRNA injection reduces the feeding response} to sucrose in vivo

Three groups of snails injected with nNOS dsRNA $(n=19)$, nNOS ssRNA $(n=19)$, or saline buffer $(n=16)$ were subjected to behavioral analysis. An ANOVA with repeated measures detected a significant source of difference between the sets of data obtained in the three groups $\left(F_{(2,51)}=14.4 ; p<0.001\right)$. Multiple pair-wise comparisons of the behavioral data revealed that the feeding response of the nNOS dsRNA-injected group was significantly more suppressed at all three time points compared with both the ssRNA-injected and saline-injected controls [Tukey's honestly significant difference (HSD) tests; $p<0.003$ ]. There was no significant suppression attributable to the injection of ssRNA. The ANOVA indicated that there were no time-dependent changes in the difference between the same groups, indicating that the inhibitory effect of nNOS dsRNA on feeding had apparently developed by $3 \mathrm{hr}$ and showed no sign of reversing at $48 \mathrm{hr}$ after injection (Fig. 1A). Additional statistical analysis of the saline control group revealed that at $3 \mathrm{hr}$ there was a significant suppression of feeding compared with 24 or $48 \mathrm{hr}$ (ANOVA with repeated measures, $F_{(2,30)}=4.0, p<0.03$; paired $t$ tests, $p<$ $0.05)$. Thus we cannot rule out that a nonspecific effect of the injection contributes to the reduction in the feeding response to dsRNA seen at the early time point, making an accurate determination of the onset of the dsRNA-specific effects difficult.

At $24 \mathrm{hr}$, the nonspecific injection effect is no longer a component of the suppressive response to the nNOS dsRNA (see responses to saline injection at 24 and $48 \mathrm{hr}$ ) (Fig. 1A). The suppressive response is confined to the dsRNA and there is no significant behavioral consequence of injecting ssRNA. Because dsRNA itself might be recognized as a pathogen, triggering a suppression of feeding, it was important to demonstrate that the behavioral effect is attributable to the ability of the injected dsRNA to target the nNOS mRNA. To examine this (Fig. $1 B$ ), we made a direct comparison between the feeding responses of animals injected $24 \mathrm{hr}$ previously with nNOS dsRNA $(n=10)$, an unrelated dsRNA $(n=10)$, nNOS ssRNA $(n=11)$, or saline $(n=$ $10)$. Only the injection of nNOS dsRNA had a significant suppressive effect on feeding responses (ANOVA, $F_{(3,37)}=4.8, p<$ 0.006; Tukey's HSD tests, $p<0.02$ ).

\section{dsRNA injection blocks fictive feeding in vitro}

Snails were injected either with nNOS dsRNA $(n=8)$ or with ssRNA $(n=8)$ and then dissected to create semi-intact lip-CNS preparations which were tested for spontaneous fictive feeding and for their response to sucrose applied to the lips. Fictive feeding was monitored by intracellular electrophysiological recording of the activity in motoneuron B3, an identified component of the feeding neural network. Spontaneous bursts of activity in this neuron, indicative of fictive feeding cycles, were equally $(p=0.5)$ low in both dsRNA-injected $(1.1 \pm 0.5$ cycles per $2 \mathrm{~min})$ and ssRNA-injected $(0.75 \pm 0.3$ cycles per $2 \mathrm{~min})$ groups of semi-intact preparations. However, preparations made from animals injected with dsRNA showed a significantly smaller sucroseevoked increase $(22 \pm 69 \% ; p<0.01)$ (Fig. $2 A)$ over spontaneous rates compared with preparations made from ssRNA-injected animals (341 $\pm 82 \%$ ) (Fig. $2 A$ ). Examples of intracellular recording from B3 motoneurons shown in Figure 2Bi,Bii demonstrate a number of characteristic fictive feeding cycles in response to sucrose in an ssRNA-treated preparation but not in a dsRNAtreated preparation.

\section{dsRNA injection interferes with NO synthesis}

Whole CNSs were isolated from animals injected previously with the synthetic nNOS dsRNA $(n=10)$, ssRNA $(n=11)$, or saline $(n=12)$ and then incubated with the NO indicator dye DAF-2 as 
A

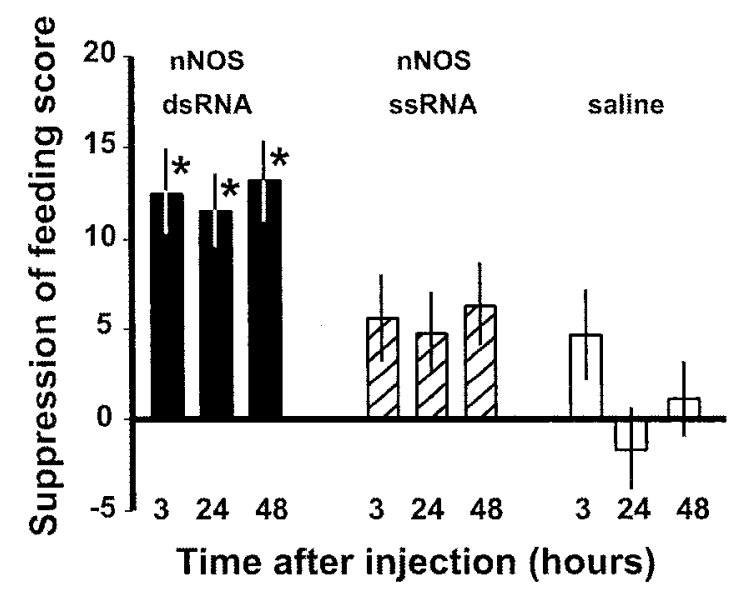

B

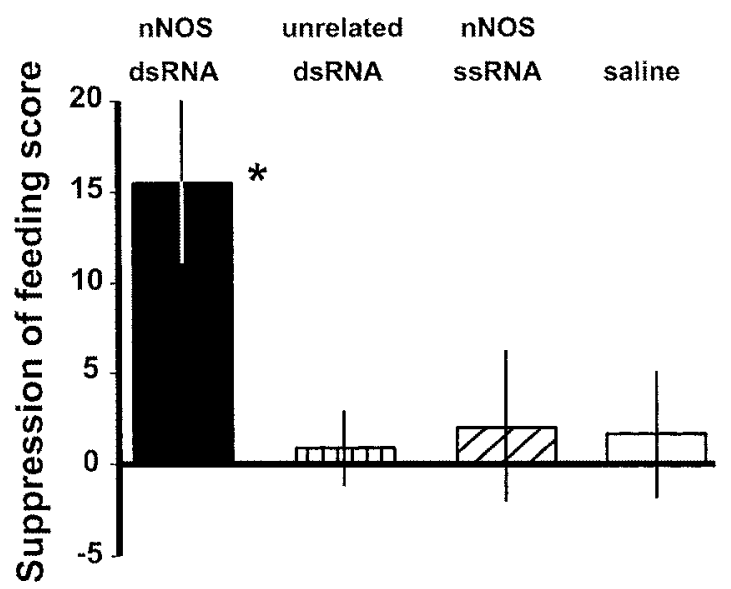

Figure 1. Injection of dsRNA targeted to Lym-nNOS mRNA causes a significant suppression in the feeding response to sucrose in vivo. A, Snails were tested for their feeding response to sucrose before they were injected with in vitro synthesized dsRNA or ssRNA or saline buffer. The animals were then retested 3,24 , and $48 \mathrm{hr}$ after the injection and the postinjection feeding score was subtracted from the preinjection score. A suppression of the feeding response is indicated by a positive difference score (suppression of feeding score). Note that suppression of feeding was significantly higher at all three time points in the dsRNA-injected group (black bars) than in either the ssRNA-injected animals (hatched bars) or saline-injected animals (white bars). However, injection with saline also caused some suppression at $3 \mathrm{hr}$ compared with 24 and $48 \mathrm{hr}$ (see Results for statistical analysis). $B$, Verification of the specificity of suppression of feeding for an nNOS dsRNA. The experiment was performed in the same way as in $A$, but an additional control group, injected with a dsRNA unrelated to nNOS mRNA (unrelated dsRNA) was used. Tests were performed at $24 \mathrm{hr}$ after injection. Only the nNOS dsRNA has a significant suppressive effect on feeding behavior. Asterisks in $A$ and $B$ indicate significant differences from controls of at least $p<0.05$. See Results for statistical details.

described above. For each sample, the emission of DAF-2 at 515 nm was measured immediately $\left(t_{0}\right)$ and then 2 hr later $\left(t_{120}\right)$. Over this time there was a significant increase in emission in the salineand ssRNA-treated snails (Fig. 3) (paired $t$ tests: saline, $p<0.05$; ssRNA, $p<0.05$ ), indicating the synthesis of NO. In contrast, there was no significant difference in the DAF-2 emission at $t_{0}$
A
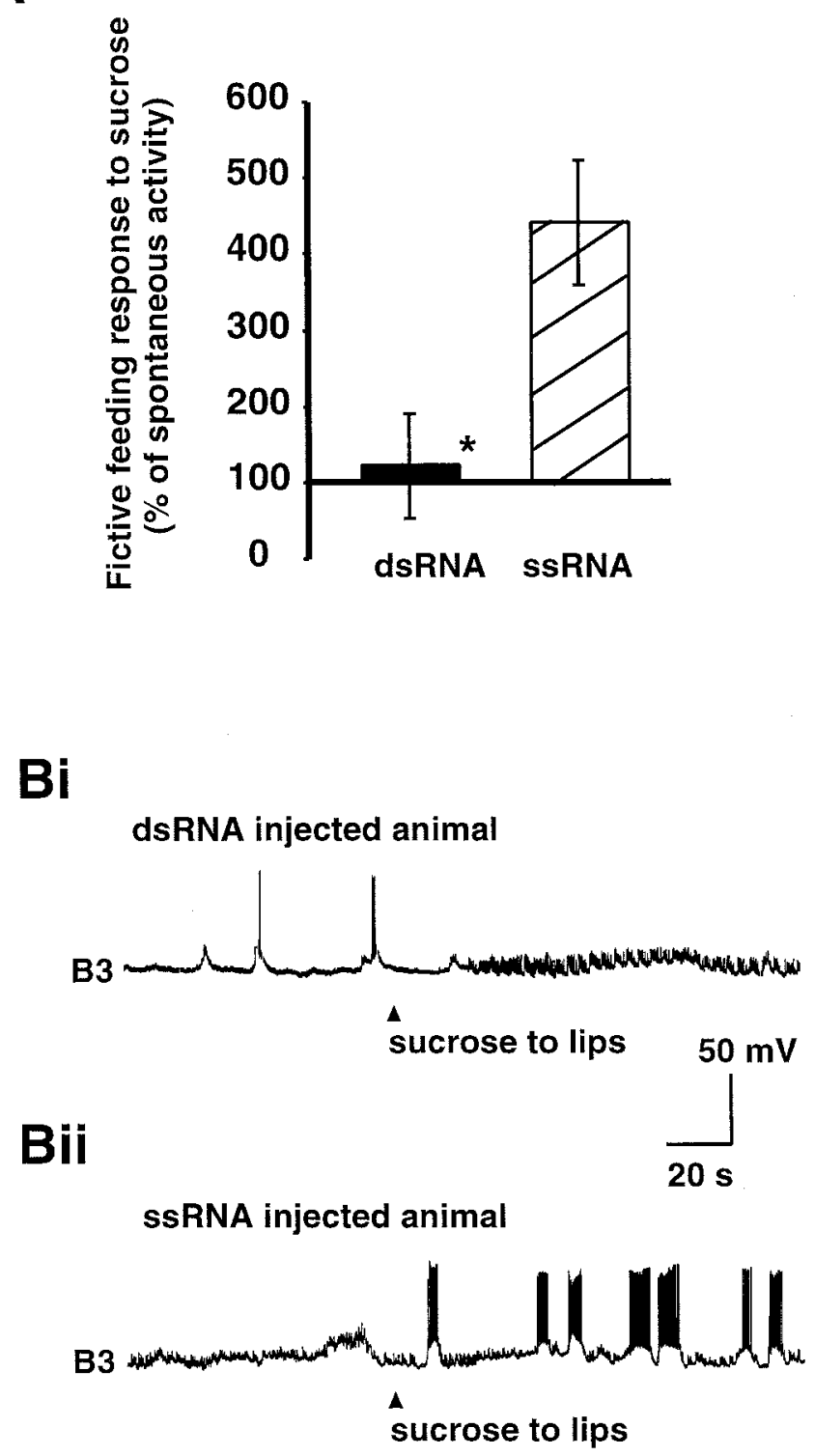

Figure 2. Suppression of fictive feeding in vitro by dsRNA injection. $A$, The number of spontaneous fictive feeding cycles of activity in the B3 feeding motoneuron were counted for $2 \mathrm{~min}$ before the application of sucrose to the lips. The percentage increase over this spontaneous rate was measured for 2 min after a sucrose stimulus in preparations made from animals injected with either dsRNA (black bar) or ssRNA (hatched bar). There is an almost complete suppression of sucrose-evoked fictive feeding in the preparations from animals injected with dsRNA. Asterisk indicates a significant difference of at least $p<0.05$. B, Sample intracellular recordings from the $\mathrm{B} 3$ motoneuron in semi-intact preparations made from dsRNA-injected $(B i)$ and ssRNA-injected (Bii) animals. Sucrose evokes prolonged fictive feeding activity in the $\mathrm{B} 3$ motoneuron in the ssRNA-treated preparation only.

and $t_{120}$ in samples prepared from snails injected with dsRNA (Fig. 3) (paired $t$ test: $p=0.67$ ). Additional comparisons revealed that the emission value at $t_{120}$ for the dsRNA-injected animals differed significantly from $t_{120}$ values for the saline- and the ssRNA-injected groups (ANOVA: $F_{(2,30)}=5.3, p<0.01$, followed by Tukey's HSD tests: $p<0.05$ for dsRNA vs ssRNA and dsRNA vs control). No significant differences were found between the $t_{0}$ values of the three groups (ANOVA: $F_{(2,30)}=2.05$, $p<0.15)$. 


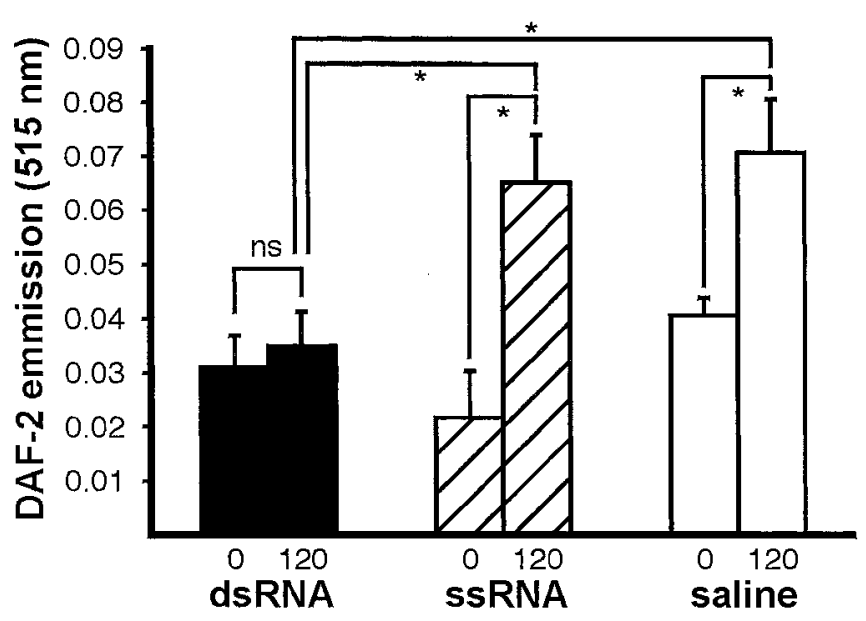

Figure 3. NO production in isolated CNSs measured with DAF-2. The isolated CNS of individual snails was incubated in a solution of the NO indicator dye DAF-2 in the presence of L-arginine. The $515 \mathrm{~nm}$ emission (excitation wavelength, $485 \mathrm{~nm}$ ) was measured for each sample immediately after adding the CNS to the solution $\left(t_{0}\right)$ and after a $2 \mathrm{hr}$ incubation at room temperature $\left(t_{120}\right)$. For the groups injected with saline buffer (white bars) or ssRNA (hatched bars), the average $515 \mathrm{~nm}$ emission increased two- to threefold during the $2 \mathrm{hr}$ incubation period. In contrast, the average $515 \mathrm{~nm}$ emission for the group of animals injected with dsRNA (black bars) showed no significant increase over the same period. Asterisks indicate significant differences of at least $p<0.05$.

\section{dsRNA injection silences the nNOS gene}

To examine the effect of dsRNA injection on gene expression, we used RT-PCR. Expression of nNOS and $\beta$-tubulin mRNAs was analyzed in three distinct groups of snails (each containing nine animals) injected with dsRNA or ssRNA or saline.

Five CNSs from each group dissected $6 \mathrm{hr}$ after the injection and four brains dissected $24 \mathrm{hr}$ after the injection were subjected individually to RT-PCR analysis. Products of the reactions were resolved in an agarose gel and the expression level of nNOS mRNA in each CNS was calculated relative to the internal standard ( $\beta$-tubulin). The data were subjected to a two-way ANOVA. This revealed a significant overall difference in the level of nNOS mRNA expression between the three groups $\left(F_{(2,26)}=\right.$ $3.51 ; p<0.05)$ but not between the two injection time points $(6$ and $24 \mathrm{hr}$ ). Multiple post hoc tests showed that the source of the difference was a significantly (Tukey's HSD tests; $p<0.05$ ) lower level of nNOS mRNA expression in dsRNA-injected (mean relative density, $0.2 \pm 0.06$ ) compared with both ssRNA- and salineinjected animals (mean relative density, $0.52 \pm 0.09$ and $0.45 \pm$ 0.11 , respectively). The ssRNA- and saline-injected groups were not different from one another $(p=0.6)$ (Fig. 4A).

Having established in groups of individual CNSs a statistically significant inhibitory effect of the dsRNA injection on nNOS gene expression, we performed an RT-PCR analysis on pooled samples containing equal aliquots from nine individual RNA preparations. The results of the experiment shown in Figure $4 B$ clearly demonstrate the decrease of nNOS gene expression in dsRNAinjected animals in comparison with those injected with either ssRNA or saline.

\section{DISCUSSION}

By exploiting the power of RNAi, we have successfully linked the expression of an nNOS gene with a specific instance of animal behavior. Importantly, we show first that the injection of nNOStargeted dsRNA molecules into intact adult snails reduces the
A

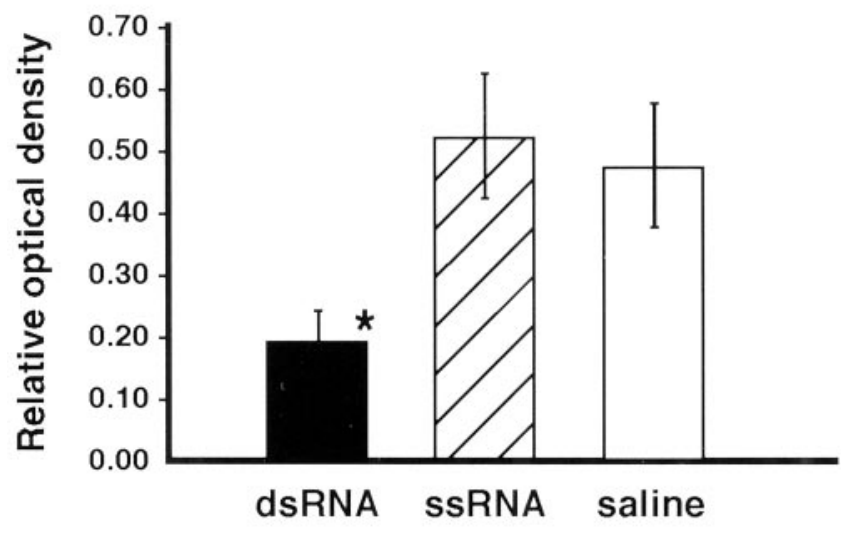

B

$1 \quad 2 \quad 3$

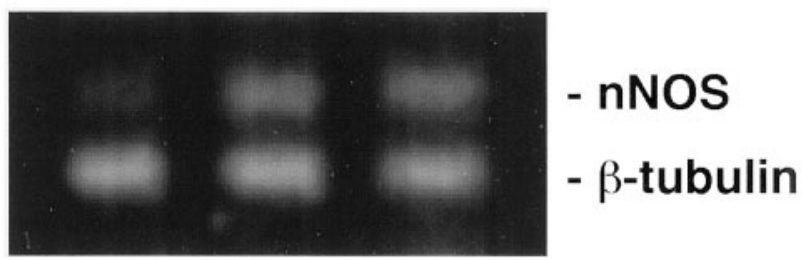

Figure 4. dsRNA injection silences the nNOS gene. $A$, CNSs dissected from animals injected with dsRNA, ssRNA, or saline were subjected individually to RT-PCR. The level of nNOS expression in each CNS was calculated relative to $\beta$-tubulin (internal control). Note that nNOS expression is significantly lower in the dsRNA-injected group (black bar) than in the ssRNA-injected (hatched bar) or saline-injected (white bar) groups. Asterisk indicates a significant difference from controls of at least $p<0.05$. $B$, The results of RT-PCR experiments on RNA pooled from the dsRNA-injected, ssRNA-injected, or saline-injected CNSs $(n=9$ in each group). Expression of nNOS and $\beta$-tubulin mRNAs was analyzed simultaneously. A marked decrease in the amount of nNOS mRNA in the dsRNA-injected group (lane 1) compared with the ssRNA-injected (lane 2) or saline-injected (lane 3) controls has been detected. Note that the level of $\beta$-tubulin mRNA expression remains very similar in all three groups.

feeding response in vivo. Second, we demonstrate that the effect of dsRNA is sufficiently robust to survive dissection, enabling us to observe the effects of RNAi in identified neurons of the feeding neural network in vitro. Third, we show that the injection of dsRNA inhibits the production of NO in the CNS. Finally, we verify that RNAi has occurred by showing that nNOS dsRNA specifically silences nNOS gene expression. Together, these findings show for the first time directly that the expression of the nNOS gene is essential for normal feeding behavior in this animal.

Until recently this result would have been extremely difficult to achieve in Lymnaea, or indeed in any other genetically intractable animal. RNAi was first discovered in the nematode Caenorhabditis elegans (Fire et al., 1998) but has now also been applied to other invertebrate species (Kennerdell and Carthew, 1998; Ngo et al., 1998). Indeed RNAi has been reported recently in the mollusc 
Aplysia, in which it has been used to support the role of the CCAAT/enhancer binding protein in long-term facilitation (Lee et al., 2001). In this example, however, the dsRNA was injected directly into neurons and gene silencing was not directly verified by showing a decrease in the levels of the target mRNA. Attempts to apply RNAi to more complex organisms such as vertebrates have generated rather controversial results. For example, initial reports demonstrating the success of RNAi (Wargelius et al., 1999; Wianny and Zernicka-Goetz, 2000) have subsequently been contradicted. In particular, experiments on a human prostate cancer cell line showed that the injection of dsRNA molecules was totally ineffective in this system (Lin et al., 2001). Furthermore, dsRNA causes nonspecific defects when used for gene silencing in zebrafish embryos (Zhao et al., 2001). Surprisingly, the question of specificity of RNAi is rarely considered and the majority of reports that claim its successful use do not show that the introduction of dsRNA molecules leads to a reduction in the amount of the targeted mRNA. It was for this reason that we considered it important to show not only that there is a specific behavioral phenotype associated with the injection of dsRNA targeted to the nNOS mRNA but also that the nNOS gene is silenced.

It is of interest to note that the RNAi-induced suppression of feeding behavior was weaker than can be achieved in pharmacological experiments in which NO depletion and NOS inhibitory drugs are used (Elphick et al., 1995; Kemenes et al., 2002). This suggests the existence of other NOS transcripts in the CNS that are involved in feeding behavior but whose expression was not affected by the dsRNA we used. In fact this is confirmed by our identification of another NOS-related mRNA, the molecular characterization of which is currently underway. Interestingly, the expression of this second transcript was not altered by the injections. This indicates a very high degree of specificity of RNAi in our system, because the two transcripts are $\sim 80 \%$ identical to one another.

In the snail, NO is not only required for the activation of feeding behavior but has been shown recently to have a crucial role in long-term memory formation after the appetitive conditioning of the feeding response (Kemenes et al., 2002). We expect therefore that the application of verifiable RNAi in intact animals, such as we have demonstrated, will significantly advance our understanding of the molecular mechanisms involved in behavioral plasticity.

\section{REFERENCES}

Benjamin PR, Elliott CJH (1989) Snail feeding oscillator: the central pattern generator and its control by modulatory interneurons. In: Neuronal and cellular oscillators (Jacklet J, ed), pp 173-214. New York: Dekker.

Bredt DS, Snyder SH (1992) Nitric-oxide, a novel neuronal messenger. Neuron 8:3-11.

Elphick MR, Kemenes G, Staras K, O'Shea M (1995) Behavioral role for nitric oxide in chemosensory activation of feeding in a mollusc. J Neurosci 15:7653-7664.

Fire A, Xu SQ, Montgomery MK, Kostas SA, Driver SE, Mello CC (1998) Potent and specific genetic interference by double-stranded RNA in Caenorhabditis elegans. Nature 391:806-811.

Garthwaite J, Boulton CL (1995) Nitric oxide signaling in the central nervous system. Annu Rev Physiol 57:683-706.

Jacklet JW (1997) Nitric oxide signaling in invertebrates. Invert Neurosci 3:1-14.

Kemenes I, Kemenes G, Andrew RJ, Benjamin PR, O'Shea M (2002) Critical time-window for NO-cGMP-dependent long-term memory formation after one-trial appetitive conditioning. J Neurosci 22:1414-1425.

Kennerdell JR, Carthew RW (1998) Use of dsRNA-mediated genetic interference to demonstrate that frizzled and frizzled 2 act in the wingless pathway. Cell 95:1017-1026.

Korneev SA, Piper MR, Picot J, Phillips R, Korneeva EI, O’Shea M (1998) Molecular characterization of NOS in a mollusc: expression in a giant modulatory neuron. J Neurobiol 35:65-76.

Korneev SA, Park JH, O'Shea M (1999) Neuronal expression of neural nitric oxide synthase (nNOS) protein is suppressed by an antisense RNA transcribed from an NOS pseudogene. J Neurosci 19:7711-7720.

Lee JA, Kim HK, Kim KH, Han JH, Lee YS, Lim CS, Chang DJ, Kubo T, Kaang BK (2001) Overexpression of and RNA interference with the CCAAT enhancer-binding protein on long-term facilitation of Aplysia sensory to motor synapses. Learn Mem 8:220-226.

Lin SL, Chuong CM, Ying SY (2001) A novel mRNA-cDNA interference phenomenon for silencing bcl-2 expression in human LNCaP cells. Biochem Biophys Res Commun 281:639-644.

Moroz LL, Park JH, Winlow W (1993) Nitric oxide activates buccal motor patterns in Lymnaea stagnalis. NeuroReport 4:643-646.

Ngo H, Tschudi C, Gull K, Ullu E (1998) Double-stranded RNA induces mRNA degradation in Trypanosoma brucei. Proc Natl Acad Sci USA 95:14687-14692.

Wargelius A, Ellingsen S, Fjose A (1999) Double-stranded RNA induces specific developmental defects in zebrafish embryos. Biochem Biophys Res Commun 263:156-161.

Wianny F, Zernicka-Goetz M (2000) Specific interference with gene function by double-stranded RNA in early mouse development. Nat Cell Biol 2:70-75.

Zhao ZX, Cao Y, Li M, Meng AM (2001) Double-stranded RNA injection produces nonspecific defects in zebrafish. Dev Biol 229:215-223. 